\title{
Routing Schemes for Wireless ATM Networks
}

\author{
A. Hameed ${ }^{1}$ and Ali Oudah ${ }^{2}$ \\ Faculty of Manufacturing Engineering \\ Pekan, 26600 \\ University Malaysia Pahang \\ ${ }^{1}$ multicore.processor@yahoo.com, ${ }^{2}$ mark6ge@gmail.com
}

\begin{abstract}
In this paper, the Wireless ATM rerouting procedures are analyzed and categorized based on a standard network topology, derived from the Wireless ATM reference model. A new operational concept for a mobile ATM network called Mobile Network Architecture based on Virtual Paths (MNA VP), in which the network nodes are connected to each other via preestablished permanent virtual path connections with fixed capacity assignments is being proposed and described. Finally, the handover hysteresis concept is introduced and a hysteresis gain is defined and calculated as the factor by which the handover rate is reduced through the use of the hysteresis.
\end{abstract}

Keywords: Wind energy, active control, energy capture, variable speed wind turbines

\section{Introduction}

Over the last few years, one of the major commercial successes in the telecommunications world has been the widespread diffusion of cellular mobile telephone services, whose provision relies on sophisticated algorithms implemented by state-of-the-art dedicated computer equipment. Lately, the challenge resides in upgrading the service offer to mobile users to include high-speed data communication services. A natural approach in this direction is to adopt the Asynchronous Transfer Mode (ATM) in the wireless environment, resulting in the so-called wireless ATM (WATM) network. However, ATM was developed for fixed networks and mobility management functionality had to be added to the traditional set of capabilities. Mobile or Wireless ATM consists of two major components: the radio access part which deals with the extension of ATM services over a wireless medium and the mobile ATM part which addresses the issue of enhancing A TM for the support of terminal and service mobility in the fixed portion of the WATM network. Wireless ATM started as a technology designed to be used for LAN or fixed wireless access solutions, where low mobility constraints are encountered. Further research projects and standardization activities coordinated by the ATM Forum demonstrated the feasibility of broadband radio access networks based on ATM technology, which can offer full-scale mobility together with all the range of ATM service capabilities existent also in the fixed ATM networks [8].

Mobility management has two distinct components: location management dealing with the correspondence between the subscriber's data and his current location and handover management which controls the dynamic re-routing and transfer of connection for the terminals crossing cell boundaries.

The frequency-domain supposed to be used for Wireless ATM, situated in the Ghz range, will imply the existence of small size cells, to cope also with the increased demands regarding system capacity. This will lead, in conjunction with a higher terminal mobility, to a very large 
number of hand over of virtual connections. Furthermore, smaller cells have tighter delay constraints, as the overlapping distances of the cells are smaller. The more complex handover procedure has higher requirements regarding radio resource management functions for the air interface paired with network signaling and control functions for handover control, Quality of Service (QoS) management and rerouting of the connection to the new network access point. Exactly these rerouting procedures are the subject of this paper. A new operational concept based on Virtual Paths is introduced and the principles of handover hysteresis are analyzed using a discrete Markov chain model.

\section{Wireless ATM Network Architecture}

A various number of reference architectures can be taken into account when we talk about Wireless A TM, ranging from simple mobile terminals to complex systems containing mobile ATM switches built in ships, planes or satellites. One standard reference scenario contains a broadband wireless access system providing unrestricted roaming capabilities within a certain area of continuous radio coverage (Figure I). The base stations (Radio Access Point, RAP) are of picocellular size and implement the physical transport medium, multiple access control, data link control and basic radio res source management capabilities. The RAP does not necessarily have to provide ATM-based physical transport, it could use as well any other access technology, as for example CDMA, also because the error detection and correction capability of the ATM stack is typically low, since it was designed for a reliable network. For this paper, we assume though the existence of a ATM radio interface capable of transmitting A TM cells over the wireless medium. Special Mobile ATM switches (MAS) are positioned at the border of an ATM network, supporting end-system mobility by possessing the necessary extensions in the signaling and control planes to provide functions for mobility management and also connection handover.

All the RAP's associated with a particular MAS form a so called zone of continuous coverage. Terminal mobility inside a certain zone and the handover associated with it (intrazone handover) is handled locally by the MAS itself. Neighboring zones with uninterrupted radio coverage can form an area in which, at any time, a RAP can be found to hand a connection over to while the terminal is moving without restrictions. The size of such an area is not limited; it could take the size of the entire network. 


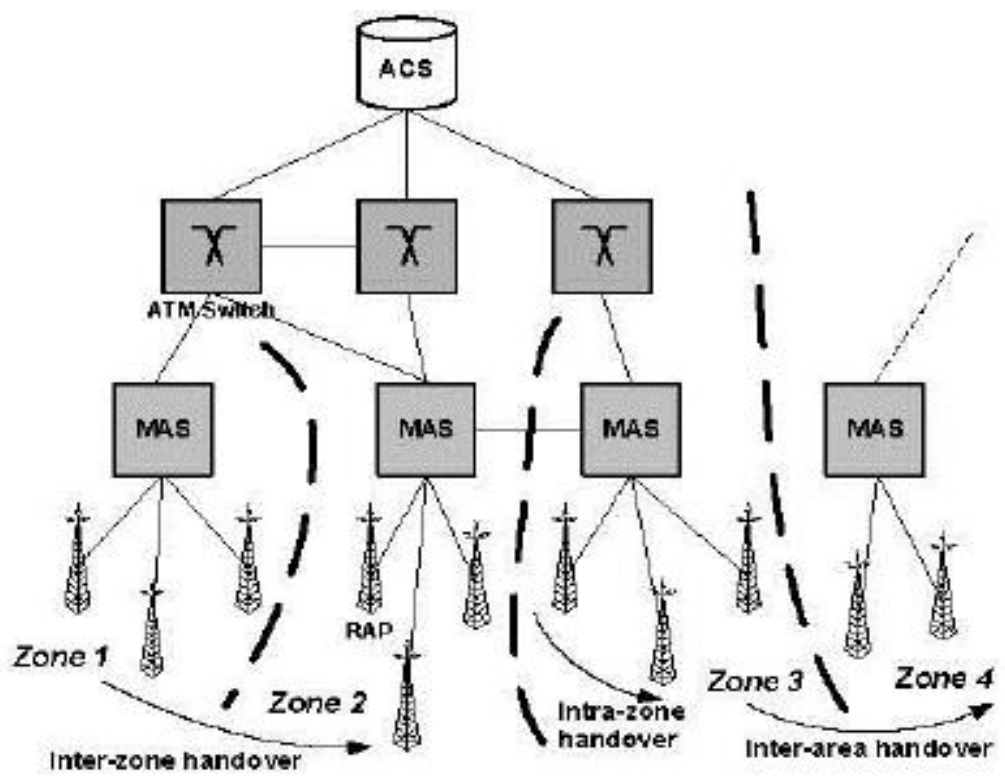

Figure 1. Architecture of the Wireless ATM Network

It is not mandatory that all the switches are able of supporting end-system mobility, therefore we introduce an hierarchically superior instance, called Area Communication Server (ACS), providing mobility control for a specific area. The ACS represents a mobility supporting A TM switch in charge of processing the protocol requests in case of a inter-zone handover. It also serves as anchor point (AP) for the active connections of the terminals inside this area. By using the ACS, the impact of the end-system mobility on the network can be significantly reduced, because there is no need any more for mobility specific functionality outside the ACS area. The disadvantage consists in the fact that connectivity cannot be guaranteed for terminals leaving this area.

A consequence of the high mobility of the terminals is the requirement of a permanent reestablishment of the virtual connection, in order to reach their current point of access to the network. This implies, beyond signaling and handover control, a process of rerouting of the connection in the A TM network. QoS control based on requirements coming from the connection itself has to be provided in order to ensure the lossless and in sequence delivery of the A TM cells during the handover process.

\section{Connection Rerouting In WATM Networks}

We can categorize the approaches for connection rerouting in four basic categories: full reestablishment, connection extension, incremental reestablishment and multicast reestablishment. They are schematically presented in Figure 2 and Figure 3, showing the connection phases during two handover steps for the different basic methods.

The most simple method is the complete reestablishment of the connection. For each change of a RAP coverage area, due to terminal mobility, a completely new VC connection is being set up between the mobile terminal and its peer. This can be done in absence of any defined handover control functions, only by the interaction of the two end systems. The major disadvantages consist in the very long duration of the procedure and the complete interruption of the service. Quite opposite to this procedure, the connection extension handover keeps the impact on a local scale. 


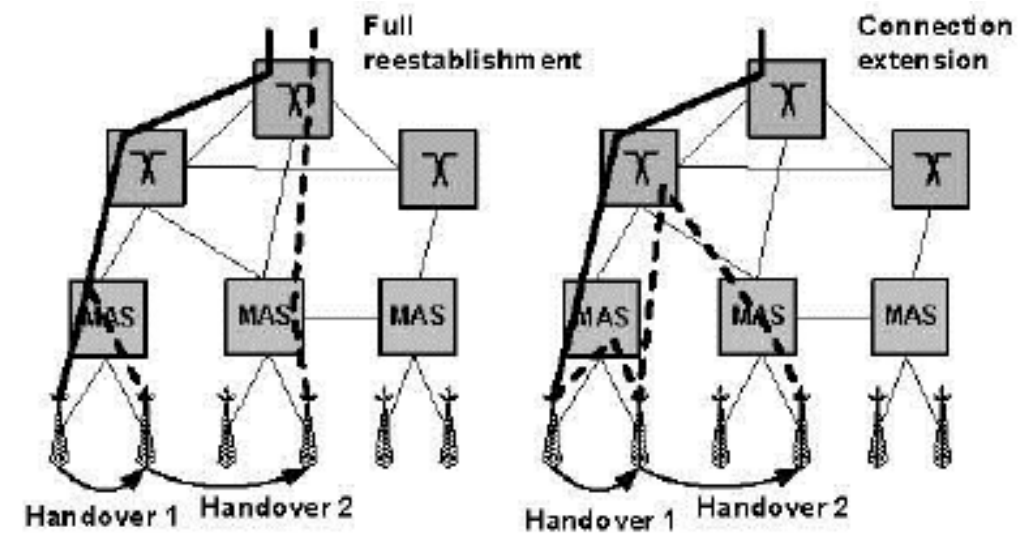

Figure 2. Connection Reestablishment and Connection Extension

Each handover is only prolonging the connection from the old RAP to the new RAP, by this achieving a very high speed, with the cost of a high routing inefficiency, due to the fact that no rerouting of the connection is performed. Loops can easily occur if the terminal is moving back and forth in a limited area, between only few neighboring cells. This method has to be combined with a routing optimization algorithm, otherwise resources are wasted. An example illustrating this scheme is presented in [9].

The multicast concept is also dealing with inefficiencies regarding the utilization of network resources. The multicast tree is established at connection setup time and can remain static or be dynamically updated for the duration of the connection $[2,16]$ All routes leading to the RAP's which will be presumly used by the mobile terminal during the connection are pre-calculated and assigned as a complete set to the connection at call setup time. This leads to a very fast handover procedure with a minimum of signaling load because no extra routing and call processing is necessary due to the preparation work done.

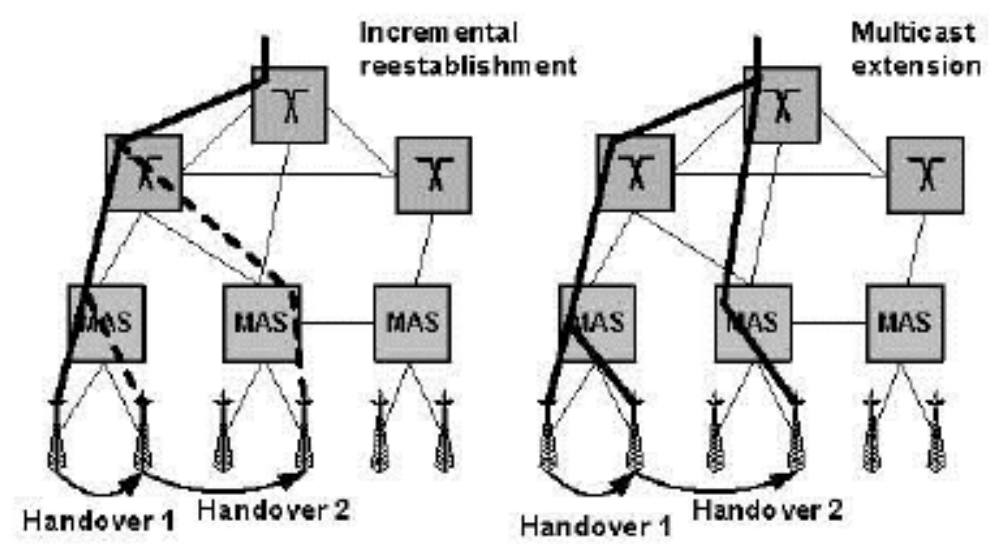

Figure 3. Incremental Reestablishment and Multicast Extension

Last but not least, the incremental reestablishment represents a more complex and therefore efficient scheme during which a rerouting decision is made for each individual handover. This decision affects only a portion of the connection, namely the one between the new RAP and some Cross Over Switch (COS) inside the current ACS area [13]. The high 
efficiency and handover speed are due to the calculation of the optimal path to the new destination RAP for each handover. The probability of reusing the longest part of the connection is quite high, which enables a fast handover, without going through the loop of routing decision.

\section{Mobile Network Architecture Based On Virtual Paths}

Basically, there are two alternatives to operate the mobile A TM network as described in Figure I: either VP-based or VC-based. We choose a VP-based mode, defining a concept called the Mobile Network Architecture based on Virtual Path (MNAVP). In the MNAVP, the MAS are net-worked with their corresponding ACS over the fixed ATM network via preestablished permanent Virtual Path Connections, as shown in Figure 4. The VPs of this architecture have fixed capacity assignments defining a virtual mobile network topology over the fixed A TM infrastructure. In this VP-based network, all intermediate A TM switches between ACS and MAS are only performing VP-switching (cross-connect functionality). Two VPCs carried on the same physical link are not statistically multiplexed. Inside a single VPC statistical multiplexing is being applied.

This virtual networking approach has several advantages. First of all, the pre-established VP topology eliminates the need for complex call routing functions and switching table updates along the VC- route, which facilitates fast handover connection setup. Second, call admission control decisions only have to be taken in the switches terminating the virtual path connections (MAS and ACS), again reducing connection setup complexity. Further, the establishment of a virtual mobile network is ideally suited for QoS-management and QoSguarantees in a multi-operator fixed network environment. The heterogeneous nature of multiservice W ATM virtual connections with a broad variety of QoS-constraints and requirements is paid attention to by separating traffic with different QoS-characteristics onto different VPs as proposed for fixed ATM networks [4], i.e., connections with similar QoSrequirements are aggregated in one VP-sub-network. Connections carrying multi-rate services can be aggregated in single VPCs, as long as they can be statistically multiplexed. Different types of services, e.g., CBR and VBR, which reduce the statistical multiplexing gain when transported together within a VPC, are separated onto different, parallel VP sub-networks.

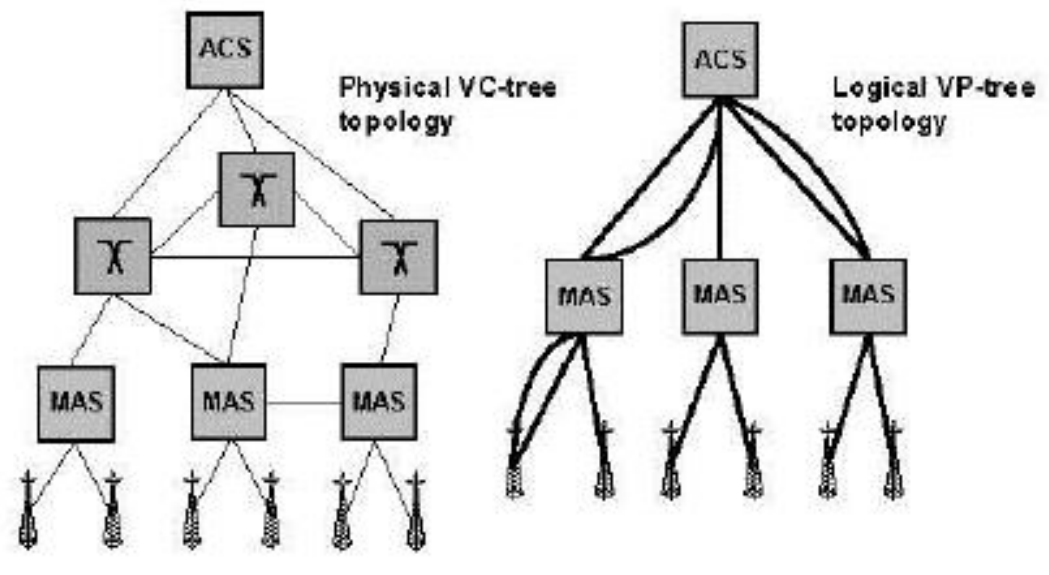

Figure 4. MN"A VP Topology

The obvious advantages of the MVPA concept are, however, achieved at the cost of losing bundling gain and a somewhat less efficient statistical multiplexing on the physical links, 
resulting in a reduced utilization of physical resources. The VP-based virtual topology networking concept in the fixed A TM subsystem is peered by VPC operated RAP-links (Figure 4), where again VPCs are used for multiservice traffic management between the MAS and the radio access points. With this two-staged approach in the MNAVP design, handovers can be handled in a partly distributed fashion, i.e., intra-zone handovers are handled locally by the MAS, whereas the ACS is involved only in inter-zone handovers. To further keep part of the handover processing within a zone, the zone can be extended virtually by the use of VPs connecting RAPs of neighboring zones to a MAS so that actually in the MNAVP virtual network the zones overlap to some extent (Figure 4, Figure 6). By that, the number of interzone handovers can be reduced, and the MAS has to do most of the work in handover call processing.

Consequently, a VC connection in a wireless ATM network consists of two different segments (Figure 5):

- $\quad$ The fixed segment from the ACS into the fixed network with the ACS operating as a Cross Over Switch (COS). This segment isn't established at call setup time and doesn't change during the lifetime of a call.

- $\quad$ The mobile segment, which follows a predetermined VP-route in the mobile network from the COS to the current RAP. This segment has to be rerouted during a connection's lifetime due to user mobility.

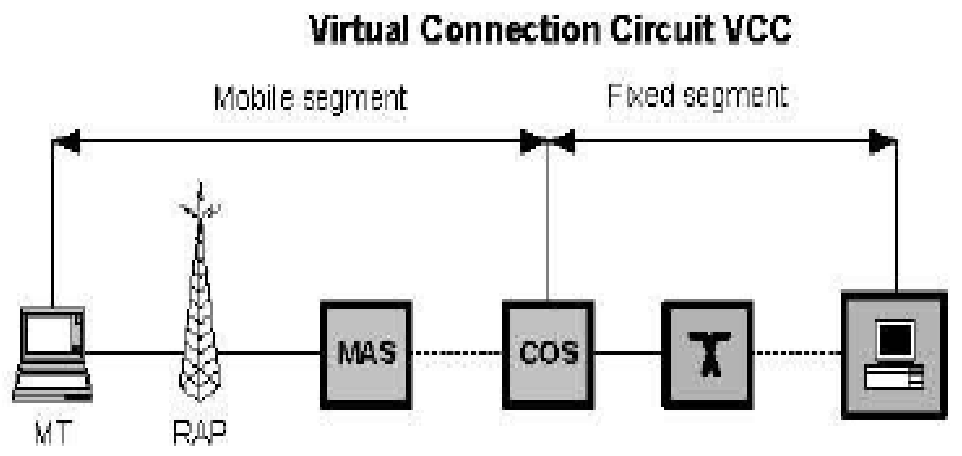

Figure 5. Fixed and Mobile Segment of a VC Connection

The most complex handover situation occurs, when the mobile moves to a zone belonging to a different ACS. This inter-zone handover situation generates the highest call processing load. The ACS responsible for the connection during call setup remains the anchoring point (COS) for that connection, switching between the fixed and mobile segment. The mobile segment has to be extended from that COS to the new RAP via the second ACS. Therefore, each ACS establishes VPCs with its neighboring ACS, reserved for carrying the inter-ACS handover connections. For best support of wide area mobility, this inter-ACS "backbone" network could have a full mesh topology. The MNA VP connection anchoring mechanism can produce non-optimal routes, which could be optimized by allowing the anchoring point COS to change during the lifetime of a connection. To reduce the number of inter-zone handover, each MAS can be virtually linked to more than one ACS. At connection setup time, one ACS has to be chosen as the connection's COS. This decision can be based on load-balancing considerations as well as mobility prediction analysis in order to minimize the number of inter-zone handover. 


\section{Handover Hysteresis in MNAVP}

The advantage of the logical network of MNAVP relies in the flexibility offered for the case of an unexpected high handover traffic between two neighbored zones, called also "anchor domains". This can be implemented by means of a VPC-based Dual Homing of the MAS at the ACS. One MAS is connected to two or more ACS via VP connection, increasing in this way the size of the anchor domain which leads in the end to an partial overlapping of those domains.

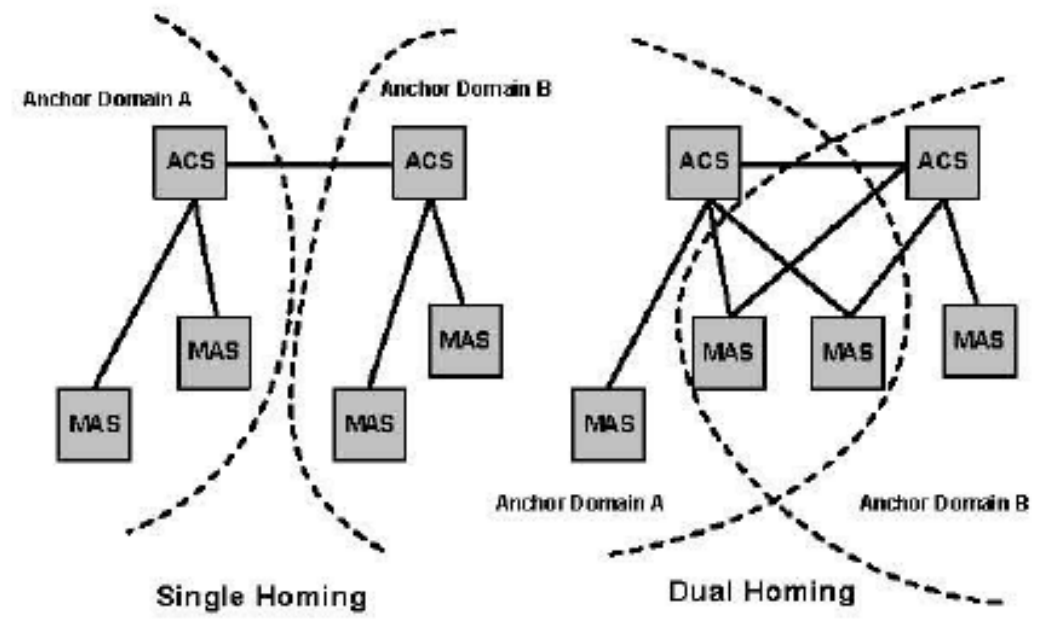

Figure 6. VP based Dual Homing

At call setup, the connection is switched through the ACS belonging to the actual physical domain. In the situation of a inter-zone handover, when the MT is crossing physical domain boundaries, it still remains in the anchor domain, due to the logical structure of the network. A rerouting of the connection over a new ACS is necessary only in the case of a repeated interzone handover. This applies also for the movement in the backward direction. By thus, a routing hysteresis which prevents the frequent occurrence of handover in case of limited geographical mobility, is introduced. The hysteresis helps preserving one of the significant advantages of the MNAVP-based rerouting procedure, namely that inside an anchor domain, the mobile segment between ACS I COS and MAS consists of only one VC segment, which would be lost in case of handover to another domain.

The analysis of the hysteresis procedure is made based on an discrete Markov chaining model. Let's consider, for the start, the case without hysteresis, shown in Figures 7 and 8. 


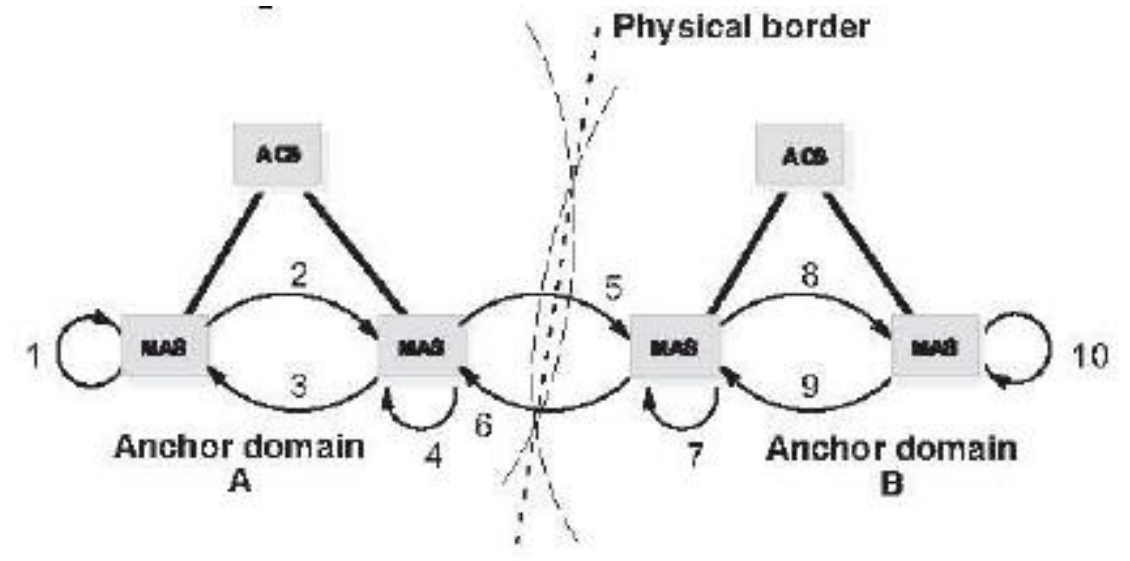

Figure 7. Handover between Neighboring Domains without Hysteresis

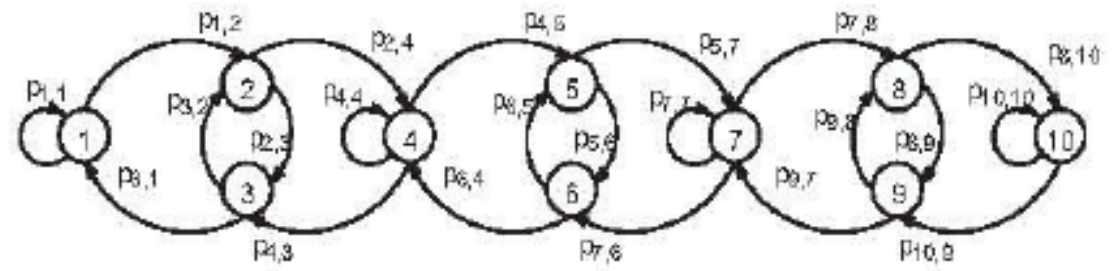

Figure 8. Discrete Markov Model for the Handover Succession without Hysteresis

This model describes the states of the different types of handover from Figure 7. The total sum of transition probabilities for one state is for all i states correspondingly

$$
\Sigma \_(\mathrm{i}-1)^{\wedge} \mathrm{n} \text { ] }
$$

The equation (2) describes the matrix of transition-states

$$
P_{H}=\left[\begin{array}{cccccccccc}
\rho_{11} & & p_{31} & & & & & & & \\
p_{12} & & \rho_{32} & & & & & & & \\
& \rho_{2,3} & & \rho_{4,3} & & & & & & \\
& \rho_{24} & & \rho_{44} & & \rho_{64} & & & & \\
& & & \rho_{4,5} & & \rho_{6,5} & & & & \\
& & & \rho_{56} & & \rho_{7,6} & & & \\
& & & \rho_{57} & & \rho_{7,7} & & \rho_{8,7} & \\
& & & & & \rho_{7,8} & & \rho_{8,8} & \\
& & & & & & p_{8,8} & & p_{109} \\
& & & & & & & p_{8,10} & & p_{1010}
\end{array}\right]
$$

To be able to calculate the probability of occurrence of an hand over, the state-probabilities of the Markov-chain are needed

$\rho$ H. $\rho=\rho$ 
After homogenizing the equation system and converting the matrix, we obtain the equation (4)

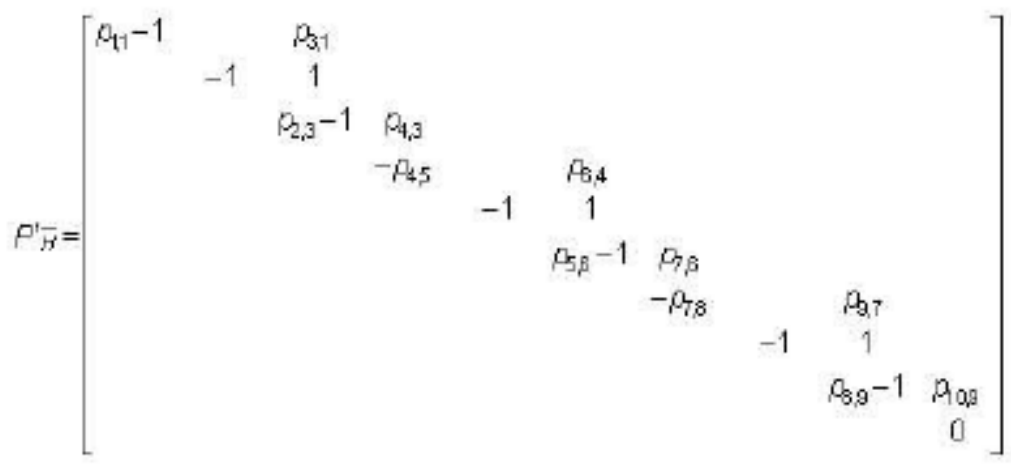

and with the condition $\mathrm{p}-\mathrm{e}=1$ for the sum of the state probabilities, the equation is

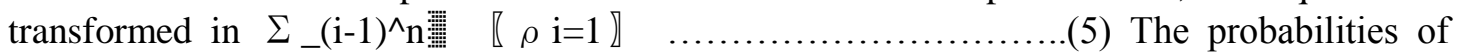
assuming the states 5 and 6 (corresponding to the inter-domain handover cases in Figure") are in fact the probabilities of occurrence of this handover type,

In the case of a handover hysteresis, the handover sequence depends upon the network nodes involved, as shown in Figure 9 and 10, The Markov model has to be extended with the corresponding states and the interzone-handover are in this case represented by the states $8 \mathrm{a}$ and $3 \mathrm{~b}$, Similar to before, we obtain the equation (6) below

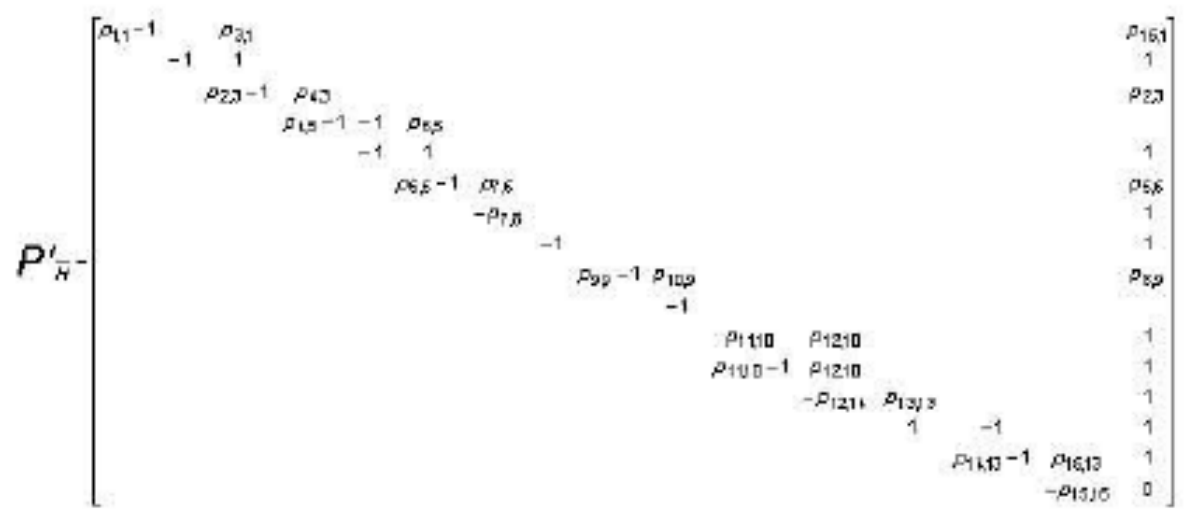

Now, the effect of the handover hysteresis can be quantified, the hysteresis gain can be defined as being the factor by which the frequency of occurrence of an inter-zone handover between anchor domains can be reduced,

$\mathrm{GH}=(\rho 5+\rho 6) /(\mathrm{p} 3 \mathrm{~b}+\rho 8 \mathrm{a})$ 


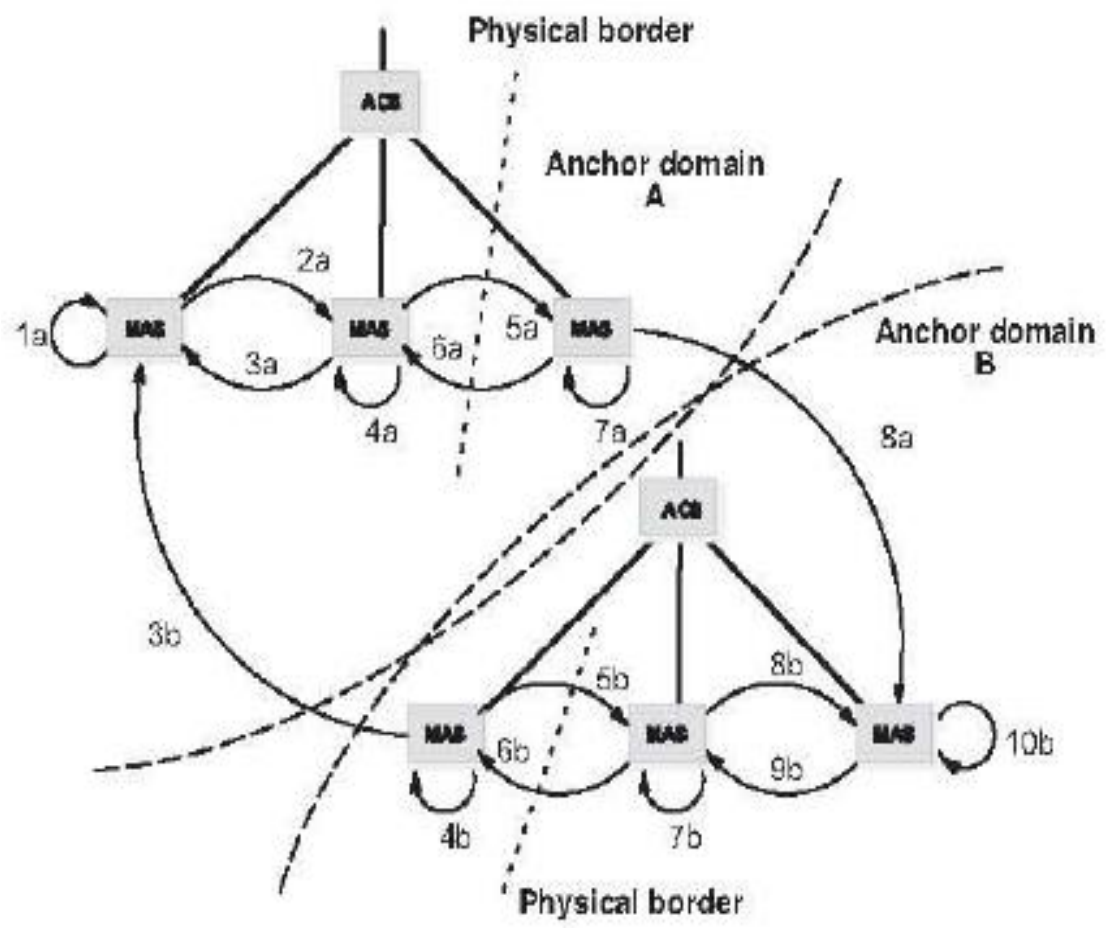

Figure 9. Handover between Neighboring Domains with Hysteresis

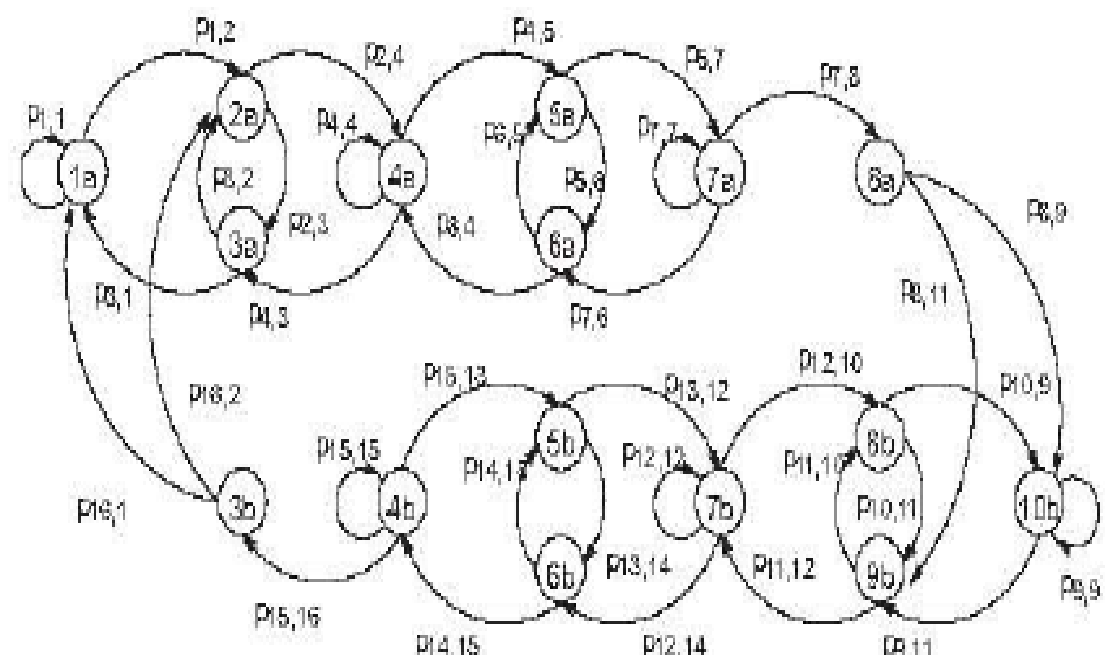

Figure 10. Discrete Markov Model for the Handover Succession with Hysteresis

The border conditions are considered symmetrical, Let's consider the random chosen variables from Table 1, which describe the local movement in terms of the probability that the MT does not leave, during it's movement, the region it belongs to, The parameter of the model with hysteresis described in Figure 8 can be determined by inserting the probabilities from Table 1 at the zone borders and, at the same time, respecting condition (1). 
Table I. Parameter for the Numerical Example of Hand Over Hysteresis

\begin{tabular}{|c|c|}
\hline Parameter & Value \\
\hline$p_{1,1}, p_{10,10}$ & 0,8 \\
\hline$p_{4,4}, p_{7,7}$ & 0,1 \\
\hline$p_{2,3}, p_{8,9}$ & 0,3 \\
\hline
\end{tabular}

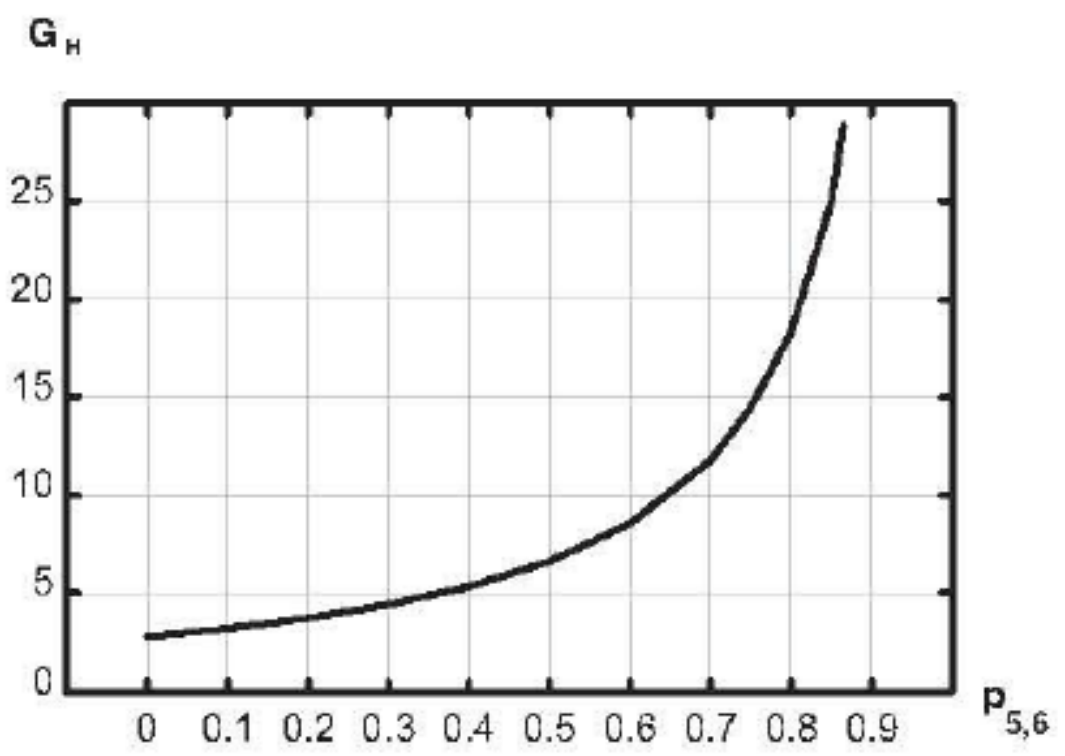

Figure 11. Hysteresis Gain

The main parameter which describes the hysteresis gain is the probability of occurrence of consecutive inter-zone handover. If this probability equals zero, the MT crosses the border, in one direction, only once. If it is high, the movement area is very restricted and the frequency of the border crossing high. Figure 11 shows this dependency and it can be observed that even in the case of a non-local movement, when PS, $6=0$, the probability of handover-occurrence is reduced to almost one third. This is based on the fact that, due to the hysteresis, one intrazone handover at the border domain (handover 4 and 7 in Figure 7) is not immediately followed by an inter-zone handover, because, as shown in Figure 9, in this case of restricted mobility area, only inter-zone handover are performed (handover $4 \mathrm{a}$ and $7 \mathrm{~b}$ ).

\section{Conclusions}

In this paper Wireless ATM reference architecture has been presented, consisting of a wireless access system coupled with the support of mobile end-systems within the ATM network. The network is subdivided in several areas served by one area server ACS, areas which are in fact handover domains providing uninterrupted radio coverage and full mobility support. After a brief description of the rerouting schemes proposed already, a new networking concept called MNA VP has been proposed, establishing a virtual mobile subnetwork over the fixed ATM infrastructure, based on pre-established virtual paths with fixed 
capacity assignments. This network design minimizes signaling and call admission load, maintaining low handover connection setup latency and facilitating high handover rates. The concept of handover hysteresis is being introduced, with the benefit of decreasing the number of inter-zone handover, for terminals showing limited geographical mobility. The frequency of occurrence of this cost-intensive handover type can be decreased with beneficial effects on the resource budget, and this is demonstrated using a Markov decision process. A hysteresis gain is defined and calculated as the factor by which the handover rate is being reduced through the use of the hysteresis.

\section{Acknowledgements}

The authors would like to thank Universiti Malaysia Pahang (UMP) for the kind help and support in conducting this research work.

\section{References}

[1] A. Acharya, B. Rajagopalan and D. Raychaudhuri, "Mobility Management in Wireless ATM Networks", IEEE Communications Magazine, vol. 35, no. 11, (1997) November.

[2] A. S. Acampora and M. Naghshineh, "Control and Quality-of $\neg$ Service Provisioning in High-Speed Microcellular Networks", IEEE Personal CommunicaJions Magazine, vol. 1, no. 2, (1994) Quarter 2.

[3] B. A. Akyol and D. C. Cox, "Rerouting for Handoffin a Wireless ATM Network", IEEE Personal Communications Magazine, vol. 3, no. 5, (1996) October.

[4] ATM-Forum: "ATM User-Network Interface Specification", The AIM Forum, Version 4.1, (2002) November.

[5] ATM-Forum: "Domain-based rerouting for active point-to $\neg$ point-calls", The AIM Forum, Version 1.0, (2001) August.

[6] K. Pierce, "Control Method for Improved Energy Capture below Rated Power", National Renewable Energy Laboratory NREL/500-26322, (1999).

[7] ATM-Forum: "Wireless Mobile TerrninallNetwork Anchor Switch Handover Model”, ATM Forum Technical Committee Wireless ATM Working Group, Contribution A TMF 97 -0265, (2003).

[8] T. S. Chen and S. S. Liu, "Management and Control Functions in ATM Switching Systems", IEEE Network, (2000) July-August.

[9] K. Y. Eng, M. J. Karol, M. Veeraraghavan, E. Ayanoglu, C. B. Woodworth, P. Pancha and R. A. Valenzuela, "BAHAMA: A broadband ad-hoc wireless ATM local-area network", ICC 95, Conference Proceedings, IEEE, (2001).

[10] M. A. Marsan, C.-F. Chiasserini, R. Lo Cigno and M. Munafo, "Local and Global Handovers for Mobility Management in Wireless ATM Networks", IEEE Personal Communications Magazine, vol. 4, no. 5, (2010).

[11] D. Minoli and T. Golway, "Planning \& Managing ATM Networks", Greenwich: Manning, (2012).

[12] H. Mitts, H. Hansen, J.; Immonen and S. Veikkolainen, "Loss less handover for wireless ATM", MONET MObile Networks and ApplicaJions, vol. 1, (2013).

[13] C.-K. T'oh, "A hybrid handover protocol for local area wireless ATM networks", MONET - MObile Networks and Applications, vol. 1, (2002).

[14] H.-J. Vogel, "Handover switching in mobile ATM networks", Conference Proceedings European Personal MObile Communications Conference EPMCC'97, Bonn, 30. Sept - 2. Okt. 1997. ITG-Fachbericht 145, Berlin, Offenbach: VDE-Verlag, 1997, S. 375-381. (2010).

[15] B. Walke, D. Petras and D. Plassmann, "Wireless ATM: Air Interface and Network Protocols of the Mobile Broadband System", IEEE Personal Communications Magazine, (2011).

[16] O. T. W. Yu and V. C. M. Leung, "Connection architecture and protocols to support efficient handoffs over an ATM / B-ISDN personal communications network", MONET $\neg$ MObile Networks and Applications, vol. 1, (2001). 